\title{
Evaluation of Mass Drug Administration Impact on Transmission of Lymphatic Filariasis in 13 Sanitary Districts in Burkina Faso
}

\author{
Nomtondo Amina Ouedraogo ${ }^{1^{*}}$, Sidnoma Muriel Ouedraogo ${ }^{1}$, Gilbert Patrice Tapsoba ${ }^{1}$, \\ W. Roland Bougma ${ }^{2}$, Mamadou Serme ${ }^{3}$, Apollinaire Kima ${ }^{3}$, Nadia Kaboret ${ }^{4}$, Ibrahim Sangare ${ }^{3}$, \\ Clarisse Bougouma ${ }^{3}$, François Drabo ${ }^{3}$
}

${ }^{1}$ Research and Training Unit in Health Sciences, University Ouaga I Pr Joseph Ki-Zerbo, Ouagadougou, Burkina Faso

${ }^{2}$ Neglected Tropical Diseases National Program, Lymphatic Filariasis Unit, Ouagadougou, Burkina Faso

${ }^{3}$ Neglected Tropical Diseases National Program (NTD NP), Ouagadougou, Burkina Faso

${ }^{4}$ University Hospital of Tengandogo, Ouagadougou, Burkina Faso

Email: *nomtondo2000@yahoo.fr

How to cite this paper: Ouedraogo, N.A., Ouedraogo, S.M., Tapsoba, G.P., Bougma, W.R., Serme, M., Kima, A., Kaboret, N., Sangare, I., Bougouma, C. and Drabo, F. (2018) Evaluation of Mass Drug Administration Impact on Transmission of Lymphatic Filariasis in 13 Sanitary Districts in Burkina Faso. Journal of Cosmetics, Dermatological Sciences and Applications, 8, 185-194.

https://doi.org/10.4236/jcdsa.2018.84020

Received: August 17, 2018

Accepted: October 19, 2018

Published: October 22, 2018

Copyright $\odot 2018$ by authors and Scientific Research Publishing Inc. This work is licensed under the Creative Commons Attribution International License (CC BY 4.0).

http://creativecommons.org/licenses/by/4.0/

\begin{abstract}
Introduction: Lymphatic filariasis (LF) is one of the neglected tropical diseases targeted for elimination as a public health issue in the world by 2020 . To achieve this goal, one of the strategies is interruption of transmission using chemotherapy (mass drug administration). Burkina Faso in west Africa, an endemic country, has endorsed this resolution. In 2000, all Sanitary Districts (SD) in the country were endemic, and then a national elimination program has made it possible to stop mass treatment in 45 out of 70 SD over 12 years. Sixteen years later, 13 other SD were reeligible for Transmission Assessment Surveys (TAS). This study aimed to determine the current level of the Mass drugs Administration (MDA) impact indicators in these 13 targeted SD in order to decide whether we need to continue MDA. Method: It was a descriptive study that took place from June to September 2017 in 13 SD regrouped into 7 Evaluation Units (EU). The population of the study was 6 to 7 years old children. The community-based cluster survey method was used. The diagnostic test used to detect Wuchereria bancroftis circulating antigen ( W. bancrofti) is the Filariasis Test Strip (FTS). The critical threshold of positivity was set at 18 positive subjects per EU. Results: We sampled a total of 12,060 children, $48.9 \%$ were female and $51.1 \%$ male. In the $7 \mathrm{EU}, 13$ children were positive to $W$. bancrofti test. The average antigenic prevalence was $0.11 \%(13 / 12,060)$. In all EU, the number of positive subjects was below the critical threshold. Conclusion: At the end of this evaluation of the transmission of lymphatic filariasis in 13 SD of Burkina Faso, we can tell the MDA
\end{abstract}


could be stopped in these areas without risk of resurgence of the disease, according to the current recommendations of WHO. This can be possible by setting up post-TMM surveillance, considering migratory flows, and including villages where positive cases were found to search possible residual transmission zones.

\section{Keywords}

Transmission Assessment Survey (TAS), Mass Drug Administration, Filariasis Test Strip

\section{Introduction}

Lymphatic Filariasis (LF) is a neglected tropical disease with vector-borne transmission. It is one of the diseases targeted by the World Health Organization (WHO) for elimination as a public health problem in the world by 2020. LF is endemic in 73 countries and about 1.39 billion people are at risk of infestation [1]. It is a parasitic disease that causes disabilities due to its chronic manifestations such as hydrocele, lymphoedema and elephantiasis. That is why LF is one of the leading causes of preventable disabilities in the world [1] [2]. Wuchereria bancrofti, Brugia malayi and Brugia timoris are the three parasitic species responsible for the disease. These parasites are transmitted to humans by the bite of mosquito: anopheles, culex, or aedes, depending on the zone. The interruption of transmission to prevent morbidity, as well as the management of the disabilities of those affected by the disease, are two components of WHO's elimination plan for filariasis. In order to stop transmission, WHO recommends the dispensation of combinated two drugs (ivermectin + albendazole) to all populations at risk in countries where there is co-endemicity with onchocerciasis. This strategy is known as "Mass Drug Administration (MDA)". MDA is a modality of preventive chemotherapy in which antihelminthic medicines are administered to the entire population of an area (e.g. state, region, province, district, subdistrict, village) at regular intervals, irrespective of the individual infection status.

The interruption of transmission involves four steps: mapping, MDA, postMDA monitoring, and checking of elimination by Transmission Assessment Surveys (TAS) [2].

Burkina Faso, in West Africa, a LF endemic country, has endorsed this elimination resolution. In 2000, a map showed endemicity of all Sanitary Districts (SD) in the country. A national elimination program was then implemented in 2001. Since then, over 124,911,968 million treatments have been administered to the population and that enabled the discontinuation of MDA in 45 out of $70 \mathrm{SD}$ in 12 years [3] [4]. Sixteen years later, 13 other SD were eligible for this assessment. Have these districts also been able to reduce the prevalence of infection to a level where resurgence of the disease is unlikely, even without mass treatment? 
The aim of this study was to determine the current level of MDA impact indicators in the 13 targeted SD in order to decide whether the mass treatment should be discontinued or not.

\section{Method and Patients}

We carried out a descriptive cross-sectional study that ran from June to September 2017 in 13 SD of the country that met the eligibility criteria of the WHO transmission assessment.

The requirements for inclusion of the SD and establishment of the evaluation units (EU) were:

- To have completed at least 5 rounds of MDA with coverage rates greater $(\geq)$ than $65 \%$. The epidemiological coverage of medicines is the proportion of inhabitants of the evaluation unit (EU) who ingested the drugs on the total population of the evaluation unit.

- To have a prevalence of microfilaraemia (Mf) less than $1 \%$ or a prevalence of filarial antigenemia (Ag) less than 2\% after the last effective MDA session for each sentinel site and point control site. The sentinel site and the control site must have $\mathrm{Mf}<1 \%$ or $\mathrm{Ag}<2 \%$ prevalence at all sites after the last successful MDA round. Plus, at least six months should have been elapsed since the last MDA. Table 1 shows the criteria used for the inclusion of SD.

The 13 SD have been regrouped into 7 Evaluation Units (EU) to meet the WHO criteria for evaluation below. All areas of an EU must share the same epidemiological features and a similar dynamic of LF transmission (epidemiological drug coverage, baseline prevalence, Mf or Ag prevalence at sentinel sites, point control sites, major LF parasites, and vector abundance). The population of the EU must not exceed two million inhabitants.

\subsection{Sampling}

The study population was 6 to 7 years-old children living in these EU. These children was born when MDA where already established. The positivity of the

Table 1. Evolution of LF prevalence in EU (Evaluation Unit).

\begin{tabular}{|c|c|c|c|c|c|}
\hline EU & $\begin{array}{l}\text { Mapping } \\
\text { prevalence } \\
\text { in } 2000\end{array}$ & $\begin{array}{c}\text { Baseline } \\
\text { Microfilaremia } \\
\text { Prevalence }\end{array}$ & $\begin{array}{c}\text { Mid-term } \\
\text { microfilaremia } \\
\text { Prevalence }\end{array}$ & $\begin{array}{c}\text { Pre-TAS } \\
\text { Prevalence }\end{array}$ & $\begin{array}{l}\text { Number of } \\
\text { actual MDA } \\
\text { rounds }\end{array}$ \\
\hline EU1: Bogandé-Manni & $52 \%$ & $23.90 \%$ & $1.80 \%$ & $0.86 \%$ & 13 \\
\hline EU2: Gayéri-Pama & $56 \%$ & $23.90 \%$ & $1.80 \%$ & $0.60 \%$ & 13 \\
\hline EU3: Garango-Pouytenga & $58 \%$ & $12.70 \%$ & $0.80 \%$ & $0.80 \%$ & 14 \\
\hline EU4: Centre 3 & $60 \%$ & $3.80 \%$ & $0.80 \%$ & $0 \%$ & 11 \\
\hline EU5: Sebba & $60 \%$ & $4.80 \%$ & $2.60 \%$ & $0.30 \%$ & 11 \\
\hline EU6: Kombissiri & $52 \%$ & $13.27 \%$ & $3.8 \%$ & $0.98 \%$ & 13 \\
\hline EU7: Manga & $46 \%$ & $16.80 \%$ & $8.80 \%$ & $0.30 \%$ & 13 \\
\hline
\end{tabular}


antigen test would be more indicative of a recent transmission of filarial infection than in adults who had previously been exposed to infection during their lifetime. Recruitment could be done in schools if the net school enrollment rate is higher than $75 \%$, if not, it should be done in the community. Due to the low rate of schooling $(<75 \%)$ in all evaluation units, we have recruited children in the community [2]. A cluster survey was conducted in each EU to form the sample.

\subsection{Selection of Clusters}

The enumeration areas (EA) from the last general population and housing census were retained as clusters. The Microsoft Excel Computer Tool, Survey Sample Builder (SSB) was used to randomly generate numbers for the chosen clusters of EA in the selected EA. SSB isused to automate the calculations for determining the appropriate clusters [5].

\subsection{Household Selection}

All the households in the selected EAs were enumerated and numbered by the head nurses of the corresponding health facilities in a census book. Only households with numbers corresponding to the numbers provided by the SSB tool were surveyed.

\subsection{Selection of Children}

All children aged 6 to 7 present in the selected household and living in the region for at least 6 months were recruited in the study. They were subjected to a blood test for filarial antigenemia. The qualitative detection of circulating antigen of $W$. bancrofti was performed on a blood sample using the Filariasis Test Strip (FTS), which is recommended by WHO for its better sensitivity [6]. All Positive results had been confirmed by at least one additional assay.

\subsection{Data Collection and Analysis}

Data collection was ensured in each EU by a team of 4 investigators including a biomedical technician. Investigators were first trained in the use of the data collection tool and the technicians were trained to use the FTS tape.

The data collection was done by a survey, including socio-demographic and biological items of the children. Additional information (length of stay in the region and level of schooling) was collected from children with a positive result.

The data were entered with the Epi Info Version 7.2 software and analyzed by IBM SPSS Statistic software version 22.

During implementation, the teams were supervised on the spot with a supervision tool [6].

\subsection{Interpretation of Results}

The threshold value is the infestation prevalence threshold below which it is 
considered that transmission cannot be continued and upsurge is unlikely even in without of MDA. This value is expressed in number of positive cases for antigens or antibodies:

- If the number of positive cases is equal or lower than to the set threshold, the EU fulfills the terms and the public authorities can decide to stop the MDA.

- If the number of positive cases is greater than the set threshold, at least two additional rounds of MDA should be performed.

The SSB tool was used to select the threshold value of 18 positive cases for each EU in this study [5]. This number is determinated automatically by the Survey Sample Builder (SSB) taking into account the sample size and the prevalence of microfilaraemia.

\subsection{Ethical Considerations}

The study protocol was submitted and approved by the ethics committee of the Ministry of Health. Consent was required from parents or the responsible adult before the inclusion of children in the study. Treatment with ivermectin and albendazole was administered free of charge to the children who were positive and to their immediate entourage.

\section{Results}

The 13 SD selected to participate to this study were Bogandé, Manni, Gayéri, Pama, Center 3 (regrouping the rural parts of SD of Boulmiougou, Nongre Massom and Signoghin), Garango, Pouytenga, Sebba, Kombissiri, Saponé, and Manga.

These SD were divided into seven evaluation units (Figure 1). The EU were subdivided into 210 community clusters in which 84 interviewers collected the data.

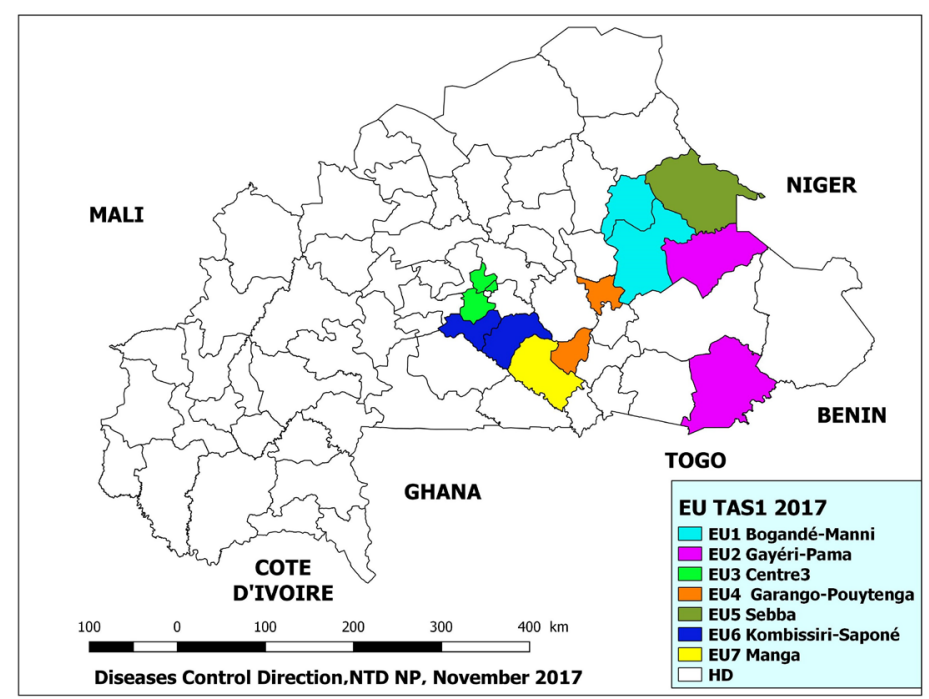

Figure 1. Location of evaluation unit (EU) during the Transmission Assessment Surveys (TAS). 
A total of 12,060 children from 6 to 7 years were recruited (Table 2). The average age of the respondents was 6.51 years old. The male subjects represented $51.06 \%$ and the female subjects $48.9 \%$. The average proportion of children submitted at least once to MDA was $77.90 \%$, from $64.21 \%$ to $89.08 \%$ respectively in Kombissiri and Sebba.

In all $7 \mathrm{EU}, 13$ children were tested positive on 12060 children, that means an average of $0.11 \%$ for filarial antigen prevalence of children born during MDA. The number of children tested positive was below the critical threshold (18 positives) in each EU. Table 3 shows the distribution by EU. In Bogande-Manni and Sebba EU, no child was tested positive for the filarial antigen. The highest number of positive children, 4, was recorded in the EU of Manga: prevalence of $0.24 \%$.

Among the 13 children tested positive, 6 were female, 7 were 6 years old. Eight out of 13 children were not in school yet (Table 4). It is mentioned that two EU (Kombissiri and Saponé) children who were tested positive came from Côte d'Ivoire (a country bordering Burkina Faso) five years earlier.

Table 2. Specifications of respondants $(n=12,060)$.

\begin{tabular}{cc}
\hline Characteristics & Size n Percentage (\%) \\
Sex & $5902(48.9)$ \\
F & $6158(51.1)$ \\
M & \\
Age & $5902(48.9)$ \\
6 & $6158(51.1)$ \\
7 & \\
Treated before & $9395(77.9)$ \\
Yes & $2614(21.7)$ \\
No & $51(0.4)$ \\
Unknown
\end{tabular}

Table 3. Microfilaraemia prevalence with FTS $(\mathrm{n}=12,060)$.

\begin{tabular}{cccc}
\hline EU & Size & Positive & Prevalence (\%) \\
\hline EU1 & 1864 & 0 & 0 \\
EU2 & 1572 & 2 & 0.13 \\
EU3 & 1702 & 2 & 0.12 \\
EU4 & 1835 & 3 & 0.16 \\
EU5 & 1795 & 0 & 0.00 \\
EU6 & 1604 & 2 & 0.12 \\
EU7 & 1688 & 4 & 0.24 \\
Total & 12,060 & 13 & 0.11 \\
\hline
\end{tabular}


Table 4. Specifications of positive cases $(n=13)$.

\begin{tabular}{cc}
\hline specifications & Effectif (n/13) \\
Sex & 6 \\
F & 7 \\
M & \\
Age & 7 \\
6 & 6 \\
7 & \\
Treated before & 9 \\
Yes & 4 \\
No & \\
School & 5 \\
Yes & 8 \\
No & 5 \\
\hline
\end{tabular}

\section{Discussion}

This evaluation, carried out in $13 \mathrm{SD}$ (regrouped in $7 \mathrm{EU}$ ) of Burkina Faso, shows an average filarial antigenic prevalence of children born during MDA of $0.11 \%$. The number of children tested positive, was below the critical threshold (18 positive) in each of the $7 \mathrm{EU}$, which is consistent with the forecasts of the Neglected Tropical Diseases National Program. Actually, the results of the mid-term and pre-TAS surveys were very conclusive compared to the baseline prevalence (Figure 1). For example, in the EU of Bogandé and Manni, we started from a prevalence of $23.90 \%$ to a mid-term prevalence of $1.80 \%$, then $0.86 \%$ to the Pre-TAS; at the end the prevalence was $0 \%$ to TAS. Therefore in the $7 \mathrm{EU}$, the end of MDA could be effective without the fear of resurgence of the disease, according to WHO evaluations (no resurgence of infection was reported above the critical limit value where active transmission is likely to occur). The low number of positive cases per EU ( 1 to 4$)$ reflects a significative reduction in the prevalence of infection in these EU compared to the EU that have achieved the same health survey of transmission in 2009 and 2012, during which the number of subjects tested positive reached 12 [7].

These results show the progress made in the interruption of transmission component and then, in the elimination of LF in Burkina Faso: the number of SD to be excluded from MDA in 2018 should be increased to 60 out of the total $70 \mathrm{SD}$ in the country, which represent $88.7 \%$ of the country's population [7]. The efforts of the neglected tropical diseases program should now be focused the remaining $10 \mathrm{SD}$.

For the post-MDA period, the other possibility is a resurgence of the disease in these supposedly controlled zones. These resurgences could come from imported cases (migration), or residual foci of transmission of the disease [8].

In the EU where positive cases were reported, 2 children (out of the 13) were 
immigrants from Côte d'Ivoire, a neighbouring country of Burkina Faso. This raises problems of migration, one of the factors that can threaten the success of lymphatic filariasis control activities [9]. Migration (permanent or temporary) of people seeking a job or a well-being is a common and growing fact in developing countries. Several migration scenarios that can impact LF elimination efforts may be encountered [8]:

- migration from endemic areas to non-endemic areas (rural or urban),

- migration from endemic areas to areas that have controlled the disease

- cross-border migration.

The last two cases are the ones concerning us: Migration from an endemic area to an area that controlled the disease within the country, and cross-border migration.

Burkina Faso is a migration country, the rural exodus towards the big cities, or more recently towards the mining zones. Thus, a migration of the endemic zones towards controlled areas is to consider because that could reintroduce the disease into these cleaned areas and the NTD program must be vigilant.

About cross-border migration, the first destination for Burkinabe immigrants is Côte d'Ivoire and also in the opposite direction, from Côte d'Ivoire to Burkina and is not only limited to border areas. The role of migrants in reintroducing transmission in "cleaned" areas would depend on several factors (climate, level of development of the country, etc.) [9] [10] [11] [12] [13]. The participation of vectors is also mentioned in the potentiality of the disease resurgence [9].

If the migrant arrives in an area where the vector cannot survive, the transmission is interrupted. The Persian Gulf countries are the destination of millions of workers from South and Southeast Asian countries, a very endemic region of the LF. The antigenemic prevalence of LF in immigrant workers in Kuwait is $18 \%$ to $20 \%$ and the prevalence of microfilariae is $3 \%$. However, there has been no evidence of infection of indigenous people [8]. Actually, the difficult environmental conditions for vector survival and the economic prosperity of the Persian Gulf countries make difficult the indigenous transmission and establishment of active transmission foci [8]. Plus, the ability of Aedes vectors to transmit the parasite responsible for LF is comparatively higher than that of $\mathrm{Cu}$ lex and Anopheles vector species. Thus, in countries where Aedes is a vector, infected migrants could be a greater threat. In some countries in the South Pacific region, where Aedes is a vector, a resurgence of LF has been observed from very low levels of post-evaluation infection [9] [14] whilst in Trinidad, where C. quinquefasciatus is the vector, no reintroduction of FL was seen in a 21-year study [10] [11].

The SD of Gayeri and Pama, bordering Niger and Sebba bordering Togo and Benin have however registered no positive cases. This brings the thought that the border zone can be used as transit for the migrant, who does not always settle there.

The implementation of an LF elimination program as well as intensive moni- 
toring and evaluation between the two countries is needed. Particular focus is needed in coordinating programs with health authorities of neighboring countries for better efficiency on both sides. The zones of high migration and the one that hosts the most population returning from endemic areas should be targeted, for a more regular control. We can work to make drugs available to migrants before entering in Burkina Faso.

The implementations of adequate post-MDA surveillance as well as the reinforcement of morbidity management in the concerned SD are the immediate challenges to be meet. Considering the villages that have recorded positive cases in the next surveys for the transmission of LF in these EU, will allow assessing the impact of the treatment of the positive cases and their entourage. It will also determine the existence of a residual focus of transmission.

\section{Conclusion}

At the end of this evaluation study of the transmission of lymphatic filariasis in 13 SD of Burkina Faso, we can affirm that the MDA can be interrupted in these zones without risk of resurgence of the disease, according to the current recommendations of WHO. That can be possible by setting up post-MDA surveillance, considering migratory flows, and including villages where positive cases were found to search for any residual transmission focus.

\section{Acknowledgements}

We thank the actors of Regionals Directions and Sanitary districts of Health Ministry, the investigators, Helen Keller International through the project End NTD's in Africa. Great thank to Serme Mamadou, the data manager.

\section{Conflicts of Interest}

The authors declare no conflicts of interest regarding the publication of this paper.

\section{References}

[1] http://www.who.int/neglected_diseases/mediacentre/WHA_50.29_Eng.pdf

[2] WHO Epidemiological Monitoring and Evaluation of Mass Drug Therapy in the Global Program to Eliminate Lymphatic Filariasis: Handbook for National PhaseOut Programs 2013, 10.

[3] Neglected Tropical Diseases National Program of Burkina Faso. Lymphaticfilariasis Mass Drug Administration Database 2001-2016.

[4] Neglected Tropical Diseases National Program, 2016 Annual Report.

[5] Task Force for Global Health, User Guide to Survey Sample Builder v2. http://www.ntdsupport.org/sites/default/files/uploads/docs/resources/SSB\%20User \%20Guide\%20V2.1_0.pdf

[6] Chu, B.K., Deming, M., Biritwum, N.K., Bougma, W.R., Dorkenoo, A.M., et al. (2013) Transmission Assessment Surveys (TAS) to Define Endpoints for Lymphatic Filariasis Mass Drug Administration: A Multicenter Evaluation. PLOS Neglected 
Tropical Diseases, 7, e2584.

[7] Neglected Tropical Diseases National Program of Burkina Faso. Lymphaticfilariasis Database 2009-2016.

[8] Ramaiah, K.D. (2013) Population Migration: Implications for Lymphatic Filariasis Elimination Programmes. PLOS Neglected Tropical Diseases, 7, e2079.

[9] Esterre, P., Plichart, C., Sechan, Y. and Nguyen, N.L. (2001) The Impact of 34 Years of Massive DEC Chemotherapy on Wuchereria bancrofti Infection and Transmission: The Maupiticohort. Tropical Medicine \& International Health, 6, 190-195. https://doi.org/10.1046/j.1365-3156.2001.00682.x

[10] Hadee, D.D., Tilluckdharry, C.C., Rawlins, S.C., Doon, R. and Nathan, M.B. (1995) Mass Chemotherapy with Diethylcarbamazine for the Control of Bancroftiafilariasis: A Twelve-Year Follow-Up in Northern Trinidad, including Observations on Mansonellaozzardi. The American Journal of Tropical Medicine and Hygiene, 52, 174-176. https://doi.org/10.4269/ajtmh.1995.52.174

[11] Rawlins, S.C., Siung-Chang, A., Baboolal, S. and Chadee, D.D. (2004) Evidence for the Interruption of Transmission of Lymphatic Filariasis among Schoolchildren in Trinidad and Tobago. Transactions of the Royal Society of Tropical Medicine and Hygiene, 98, 473-477. https://doi.org/10.1016/j.trstmh.2003.11.006

[12] Triteeraprapab, S. and Songtrus, J. (2001) High Prevalence of Wuchereria bancrofti Infection among Myanmar Migrants in Thailand. Annals of Tropical Medicine \& Parasitology, 95, 535-538. https://doi.org/10.1080/00034983.2001.11813666

[13] Sunish, I.P., Rajendran, R., Mani, T.R., Gajanana, A., Reuben, R., et al. (2003) Long-Term Population Migration: An Important Aspect to Be Considered during Mass Drug Administration for Elimination of Lymphaticfilariasis. Tropical Medicine \& International Health, 8, 316-321. https://doi.org/10.1046/j.1365-3156.2003.01033.x

[14] World Health Organization (2006) The PacELFway: Towards the Elimination of Lymphatic Filariasis from the Pacific, 1999-2005. WHO Publications, Geneva. 